\title{
Distributed Power Allocation in Cognitive Radio Networks under Network Power Constraint
}

\author{
Furqan Ahmed*, Olav Tirkkonen*, Alexis A. Dowhuszko*, and M. Juntti ${ }^{\dagger}$ \\ ${ }^{*}$ Dept. of Communications and Networking, Aalto University, P.O. Box 13000, FI-00076 Aalto, Finland \\ ${ }^{\dagger}$ Dept. of Communications Engineering, University of Oulu, P.O. Box 4500, FI-90014 Oulu, Finland
}

\begin{abstract}
We consider utility maximization in a multi-cell network under a total transmit power constraint, e.g. given by a cognitive radio geo-location database. The network utility in the downlink is maximized by allocating transmit powers in the network, while meeting the network-wide transmit power constraint. Distributed algorithms for allocating downlink transmit power are discussed, which involve exchange of prices that reflect interference between cells. Using primal decomposition, we present an online algorithm which guarantees that the network power constraint is met at all times. To this end, each cell adjusts its power level while taking into account the interference prices received from neighboring cells. Depending on the pricing information, a transmitter may reduce its power so that it can be used by some other transmitter. Distributed optimization enabled by the exchange of interference prices among cells results in an efficient distribution of total power among the transmitters. Simulation results illustrate that exchange of prices can yield a significant gain over non-cooperative and partially cooperative power allocation approaches in indoor small multi-cell networks.
\end{abstract}

\section{INTRODUCTION}

Foreseen shortages in spectrum for wireless services have paved the way for new paradigms that can enable an efficient utilization of the spectrum, while adhering to existing regulations and policies. Cognitive Radio (CR) has been proposed to improve spectrum utilization and enhance the efficiency of spectrum sharing systems. It does so by allowing Secondary Users (SUs) to opportunistically access the frequency band originally allocated to the Primary User (PU), when or where $\mathrm{PU}$ is inactive, or otherwise in a manner that does not disturbs the PUs.

In CR system based on Geo-Location DataBase (GLDB), see e.g. [1], a GLDB gives a SU the right to use a part of spectrum in a geographical location, constrained by a maximum transmit power. Similarly, in recently proposed Licensed Shared Access (LSA) [2], incumbents would grant licensees exclusive access to spectrum, subject to service conditions related to incumbent protection. A GLDB is a possible solution for realizing such protection. Moreover, in a GLDB CR system it is possible (and in an LSA system it is likely) that the secondary user (or LSA licensee) is a cellular network consisting of multiple transmitters. In such cases, the distribution of transmission power among these transmitters needs to be decided. This can be done, e.g., by a GLDB controlling the power density of a secondary network [3].

Different approaches for controlling the average or peak interference power caused by multiple SUs to PUs have been considered in [4]-[7]. In [4], the PU determines network-state dependent prices to the interference caused by the SUs, and it is shown that subject to these prices, selfish SUs playing a non-cooperative game converge to a Nash Equilibrium where the sum interference constraint is satisfied. An alternative formulation is considered in [5], where the sum rate of the SUs is maximized, subject to an outage-ratio of PU receivers. Similarly, in [6], the weighted sum rate maximization of Multiple-Input Multiple-Output (MIMO) SUs is discussed, subject to a total interference constraint. The approach is semidistributed - the master problem of distributing the transmit power to the SU transmitters is performed in a centralized manner, whereas the MIMO covariance optimization at the SUs is performed in a distributed manner with interference prices, subject to the power constraints given by the master solution.

Consider a CR power distribution problem where there is a secondary cellular network/LSA licensee, which is providing coverage in a limited geographic region, and is given a total transmit power constraint. The power has to be dynamically distributed among transmitters in different cells. This is particularly important in uplink transmission of secondary cellular networks, but applies equally well in downlink, when the total power constraint given to a network is smaller than the total maximum transmit power. Scaling behavior of CR networks with constraints on SU average total power and peak power has been considered in [8], without algorithmic considerations. A total power constraint as such, is similar to a total interference constraint, and the algorithms of [4]-[6] would apply for the power distribution problem, with suitable modifications. Our approach differs from [6], in that we present a completely distributed solution to the primal problem under network-wide constraint on total transmit power. Moreover, we consider coupled utility functions, as well as coupled constraints, whereas in [7] only the (interference) constraints are coupled.

In this paper, we address CR power distribution problem in a secondary cellular network. The system consists of multiple interfering cells, each consisting of multiple Mobile Stations (MSs) and a single Base Station (BS). Network utility maximization is considered subject to a network-wide total power constraint. To the best of our understanding, this problem has not been addressed in the literature-nor has a completely distributed algorithm been presented for power distribution problem in the simpler scenario of secondary transmitter-receiver pairs, or for the related total interference 
power problem. We propose distributed pricing based algorithms for allocating power to SUs, such that the network utility is maximized while the constraint on sum power of SUs is met. Both primal and dual decomposition based algorithms are considered.

The rest of paper is organized as follows: Section II describes the system model and formulation of optimization problem. Section III introduces the primal and dual decomposition concepts and discusses in detail distributed network power control algorithms for power distribution in CR networks. Section IV shows simulation results for a CR network in a WINNER office building [9], followed by a discussion on the performance of the proposed approaches. We conclude in Section V.

\section{System Model ANd Problem Formulation}

We consider a set of $\mathcal{I} \triangleq\{1, \ldots, I\}$ low-power BSs deployed in a geographical area, communicating with their MSs (users) using common bandwidth B. The set of MSs in the system is $\mathcal{L} \triangleq\{1, \ldots, L\}$, where BS $i$ serves the set of MSs $\mathcal{L}_{i} \subseteq \mathcal{L}$, with $L_{i}=\left|\mathcal{L}_{i}\right|$, and $\left\{\mathcal{L}_{i}: i \in \mathcal{I}\right\}$ denotes a partition of $\mathcal{L}$

The sum of transmit powers of all BSs is constrained by the total network power $P_{\max }$, i.e., $\sum_{i \in \mathcal{I}} p_{i} \leq P_{\max }$, where the transmit powers of BSs are denoted by the set $\mathbf{p} \triangleq\left\{p_{i}\right\}_{i=1}^{\mathcal{I}}$, with the (per BS) constraint $P_{\min } \leq p_{i} \leq P_{\max }$. Thus, for $\mathrm{BS} i$, the local constraint set is

$$
\mathcal{P}_{i} \triangleq\left\{p_{i} \in \mathbb{R}_{+}: P_{\min } \leq p_{i} \leq P_{\max }\right\},
$$

Intra-cell scheduling decisions of BS $i$ are reflected by the scheduling weights $\mathbf{w}_{i} \triangleq\left\{w_{l}\right\}_{l \in \mathcal{L}_{i}}$, where $w_{l}$ is the fraction of orthogonal resources that $\mathrm{BS} i$ allocates for MS $l \in \mathcal{L}_{i}$. We assume that each BS distributes all its resources among its MSs, so that the local constraint set is

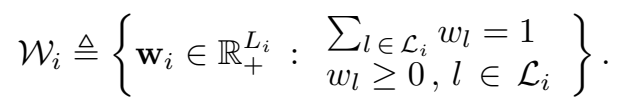

The aim is to maximize the sum-utility

$$
U_{\text {sum }}(\mathbf{p}, \mathbf{W})=\sum_{i \in \mathcal{I}} U_{i}\left(\mathbf{p}, \mathbf{w}_{i}\right)
$$

of the downlink multi-cell system, where $\mathbf{W} \triangleq\left\{\mathbf{w}_{i}\right\}_{i=1}^{I}$ is a set comprising of scheduling weights of all MSs. Here $U_{i}$ is the sum-utility of the MSs served by BS $i$

$$
U_{i}\left(\mathbf{p}, \mathbf{w}_{i}\right)=\sum_{l \in \mathcal{L}_{i}} u_{l}\left(\mathbf{p}, \mathbf{w}_{i}\right)
$$

where $u_{l}$ is the utility function of a MS $l$ served by BS $i$. The Signal-to-Interference plus Noise power Ratio (SINR) that MS $l$ (served by BS $i$ ) experiences in reception is given by

$$
\gamma_{l}(\mathbf{p})=\frac{p_{i} h_{i, l}}{I_{l}+N_{0}} \quad l \in \mathcal{L}_{i},
$$

where $h_{i, l}$ is the channel power gain between BS $i$ and MS $l$, which is assumed to be frequency flat, $N_{0}$ is the additive white
Gaussian noise power, and

$$
I_{l}=\sum_{j \neq i} p_{j} h_{j, l} \quad l \in \mathcal{L}_{i},
$$

denotes the interference experienced by MS $l$. The utility function for the users should be selected according to the performance metric to be maximized. In this paper, we consider Proportional Fair (PF)-Rate utility function [10]. This leads to fairness in sharing common resources (i.e., power and scheduling weights) among the MSs of each cell. The PF-Rate utility function is

$$
u_{l}\left(\mathbf{p}, \mathbf{w}_{i}\right) \triangleq \ln \left\{w_{l} \ln \left[1+\gamma_{l}(\mathbf{p})\right]\right\} \quad l \in \mathcal{L}_{i},
$$

which is the logarithm of the normalized Shannon rate (in nats/s/Hz), while treating interference as noise. This logarithmic form of the utility allows to improve the situation of those users that are experiencing low data-rates (due to high co-channel interference). The aim is to find the power allocation of all BSs, such that the network utility is maximized under the total network power constraint $P_{\max }$. The network level optimization problem is given by

$$
\begin{array}{cl}
\underset{\mathbf{p}, \mathbf{W}}{\operatorname{maximize}} & \sum_{i \in \mathcal{I}} U_{i}\left(\mathbf{p}, \mathbf{w}_{i}\right) \\
\text { subject to } & \sum_{i \in \mathcal{I}} p_{i} \leq P_{\max }, \\
& p_{i} \in \mathcal{P}_{i}, \mathbf{w}_{i} \in \mathcal{W}_{i}, \quad \forall i \in \mathcal{I} .
\end{array}
$$

This is a non-convex problem, for generic $U_{i}$, as the SINRs of receivers are coupled. However, it is convex for the PFRate utility that is considered here [11]. In what follows, we discuss decomposition methods that lead to pricing algorithms for finding a solution of (8) in a distributed way. Note that in frequency selective channels, this model would naturally generalize to the multichannel version of the power allocation problem, which is non-convex [12].

\section{Distributed Network Power Control}

To formulate a distributed solution to (8), primal and dual decomposition methods can be used, in conjunction with pricing algorithms for distributed optimization over $\mathbf{p}$ and $\mathbf{W}$.

\section{A. Primal Decomposition}

The primal decomposition [13] is an appropriate decomposition procedure in this case, as it can be seen that fixing power vector decouples the problem into $I$ independent scheduling weight optimization sub-problems, one per BS. Thus, the optimization problem (8) can be separated into a two level optimization procedure. At a lower level, with p fixed, we have the decoupled scheduling weight optimization sub-problems $\forall i \in \mathcal{I}$,

$$
\underset{\mathbf{w}_{i}}{\operatorname{maximize}} U_{i}\left(\mathbf{w}_{i}\right)
$$

subject to $\mathbf{w}_{i} \in \mathcal{W}_{i}$, 
which is a convex problem and can be solved at each BS. For updating the coupling variable $\mathbf{p}$, we have a master problem $\forall i \in \mathcal{I}$,

$$
\begin{array}{ll}
\underset{\mathbf{p}}{\operatorname{maximize}} & U_{i}(\mathbf{p}) \\
\text { subject to } & \sum_{i \in \mathcal{I}} p_{i} \leq P_{\max }, \quad p_{i} \in \mathcal{P}_{i} .
\end{array}
$$

Optimization over $\mathbf{p}$ couples the cells in utility function as well as in constraints. When not considering the network-wide power constraint, the problem is a conventional distributed power control problem [14]. We use a pricing algorithm to solve the optimization over $\mathbf{p}$ in a distributed way. As the utility function $U_{i}$ is continuous, this problem can be solved by an iterative descent method as discussed next.

\section{B. Components of Gradient and Pricing}

Consider the network utility in the master problem which can be written as

$$
U_{\text {sum }}(\mathbf{p} \mid \mathbf{W})=U_{i}\left(\mathbf{p} \mid \mathbf{w}_{i}\right)+\sum_{j \neq i} U_{j}\left(\mathbf{p} \mid \mathbf{w}_{j}\right) .
$$

Differentiating with respect to $p_{i}$ we have

$$
D_{i}=\frac{\partial U_{\mathrm{sum}}(\mathbf{p} \mid \mathbf{W})}{\partial p_{i}}=\frac{\partial U_{i}\left(\mathbf{p} \mid \mathbf{w}_{i}\right)}{\partial p_{i}}+\sum_{j \neq i} \frac{\partial U_{j}\left(\mathbf{p} \mid \mathbf{w}_{j}\right)}{\partial p_{i}}
$$

Let us define the following terms as power benefit and power price, respectively:

$$
\begin{aligned}
& \pi_{i i}=\frac{\partial U_{i}\left(\mathbf{p} \mid \mathbf{w}_{i}\right)}{\partial p_{i}} \quad \forall i \in \mathcal{I}, \\
& \pi_{j i}=\frac{\partial U_{j}\left(\mathbf{p} \mid \mathbf{w}_{j}\right)}{\partial p_{i}} \quad \forall j \in \mathcal{I}, j \neq i .
\end{aligned}
$$

With the PF-rate utility function, the power benefit becomes

$$
\pi_{i i}=\sum_{l \in \mathcal{L}_{i}} \frac{1}{\ln \left[1+\gamma_{l}(\mathbf{p})\right]} \frac{\gamma_{l}(\mathbf{p})}{\left[1+\gamma_{l}(\mathbf{p})\right]} \frac{1}{p_{i}} .
$$

This reflects the increase in utility $U_{i}$ per unit increase in power $p_{i}$. Likewise, the power price $\pi_{j i}$ indicates the effect of the power of $\mathrm{BS} i$ on the utility of $\mathrm{BS} j$, and is given by

$$
\pi_{j i}=-\sum_{l \in \mathcal{L}_{j}} \frac{1}{\ln \left[1+\gamma_{l}(\mathbf{p})\right]} \frac{\left[\gamma_{l}(\mathbf{p})\right]^{2}}{\left[1+\gamma_{l}(\mathbf{p})\right]} \frac{h_{i, l}}{p_{j} h_{j, l}} .
$$

By the exchange of power prices, the BSs can cooperatively maximize the network utility over their respective powers in a distributed way. Base station $i$ calculates the benefit $\pi_{i i}$ for its own use, and $\pi_{i j}$, to exchange it with each neighbor $j$. To calculate the benefits and prices terms, the BS $i$ needs to know: 1) current SINRs $\left\{\gamma_{l}\right\}_{l \in \mathcal{L}_{i}}$ of the MSs served by $i$, and the corresponding own cell channel gains $\left.\left\{h_{i, l}\right\}_{l \in \mathcal{L}_{i}} ; 2\right)$ the cross channel gains between its served MSs and the interfering BSs $\left\{h_{j, l}\right\}_{j \neq i, l \in \mathcal{L}_{i}}$; and 3) its own transmit power $p_{i}$. It is assumed that the channel gains are calculated by periodic transmission of orthogonal pilot signals by the BSs, whereas the prices are exchanged over a backhaul links that connects the BSs.

To find the solution of (8) in a distributed manner, the master problem and the secondary problems need to be solved iteratively. Compared to distributed power control, the novelty in solving (8) comes from the global constraint. It can be seen that for the PF-rate utility function, the secondary problems (i.e. optimization over $\mathbf{w}_{i}$ ) can be simplified, since closed form solution exists. For the PF-rate utility function in frequency flat static channels, the optimal scheduling weight allocation is Round Robin allocation, where all MSs get an equal share of resources. Therefore, the scheduling weights $\mathbf{W}$ are constant while the optimization over transmit powers $\mathbf{p}$ is done in an iterative manner. To do this, we devise a distributed version of the coordinate descent method on the constraint surface, following the approach given in [15], where the 1-norm is used when selecting the descent direction.

An updating BS $i \in \mathcal{I}$ receives the power prices $\left\{\pi_{j i}\right\}_{j \neq i}$, and the $\left\{D_{j}\right\}_{j \neq i}$ from all interfering BSs. With the prices available, it calculates its power benefits and constructs the $D_{i}$, which gives a complete information of how its transmit power can influence the network utility. With $\left\{D_{j}\right\}_{j \neq i}$ available for all interfering BSs, it selects a BS $j^{*}$ that can increase the network utility the most,

$$
j^{*}=\underset{j \neq}{\arg \max } D_{j}
$$

If the utility increase $D_{j^{*}}$ is larger than the own-cell increase $D_{i}$, it sends a message to that $\mathrm{BS} j^{*}$ to increase its power by a step-size $\delta$ times the difference in gradients.

To meet the total power constraint, the BS $i$ reduces its power by the same amount. To avoid excessive changes in the initialization stage, we also set a maximum absolute stepsize $\delta_{\max }$. This is followed by a power price, and $D_{i}$ update step, where BS $i$ updates its $D_{i}$ and power price $\left\{\pi_{i j}\right\}_{i \neq j}$ that corresponds to the new power profile and sends them to all interfering BSs. With this power update procedure, the network power constraint is always respected in all iterations, which makes it suitable for an online implementation. We consider asynchronous and periodic updates, so that each BS has a set of unique update times $t_{i}[n]$, where $n$ is the iteration index. Each BS updates the power, prices, and $D_{i}$ only once in an iteration. A summary of the proposed Distributed Network Power Control (DNPC) algorithm based on Primal decomposition (DNPC-Primal) is given as Algorithm 1. Note that for the PF-Rate utility function, the gradients may be unbounded. As we assume that the powers of BSs with active users are larger than a minimum power $P_{\min } \ll P_{\max }$, gradients are bounded, and the algorithm can be proven to converge. Moreover, this limitation does not change the convexity of the domain.

\section{Dual Decomposition}

To apply the dual decomposition to problem (8), additional auxiliary variables and corresponding equality constraints need to be introduced. This is essential because of the specific nature of the problem, where the coupling exists in both objective functions and the system wide constraints [16]. To this end, 


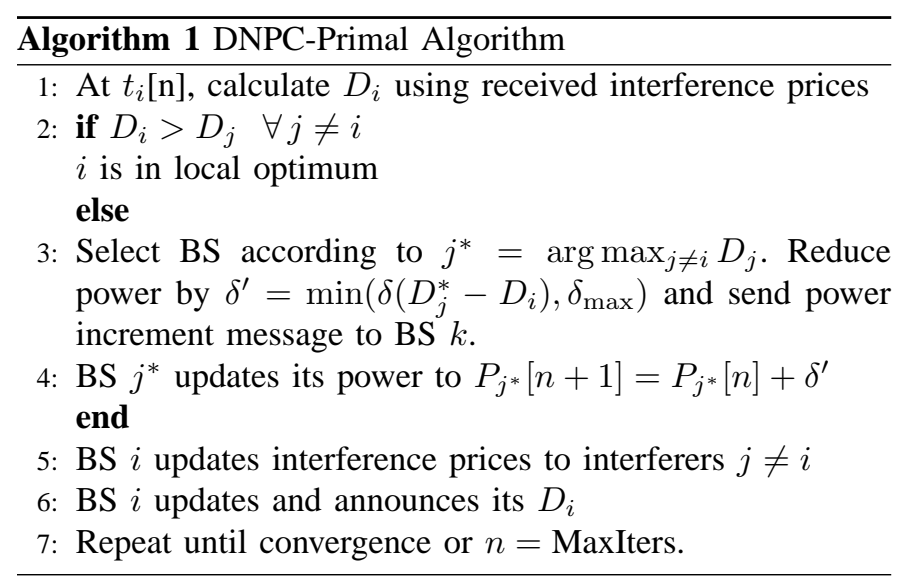

we can formalize the problem as

$$
\begin{aligned}
\underset{\left\{p_{i},\left\{p_{i j}\right\}_{j \neq i}\right\}_{i \in \mathcal{I}}}{\operatorname{maximize}} & \sum_{i \in \mathcal{I}} U_{i}\left(p_{i},\left\{p_{i j}\right\}_{j \neq i}\right) \\
\text { subject to } \sum_{i \in \mathcal{I}} p_{i} & \leq P_{\max }, \quad p_{i j}=p_{j}, \forall i, j \\
p_{i} & \in \mathcal{P}_{i}, \quad p_{i j} \in \mathcal{P}_{j}, \quad \forall i, j
\end{aligned}
$$

Note that $p_{i j}$ here is an auxiliary variable that can be interpreted as perception of BS $i$ on the power of BS $j$, which is equal to the actual power of BS $j$ at equilibrium. The equivalent form in terms of Lagrangian is given by

$$
\begin{aligned}
& \underset{i x i m i z e}{\operatorname{maximize}} \sum_{i \in \mathcal{I}} U_{i}\left(p_{i},\left\{p_{i j}\right\}_{j \neq i}\right)+\lambda\left(P_{\max }-\sum_{i \in \mathcal{I}} p_{i}\right) \\
& \left\{p_{i},\left\{p_{i j}\right\}_{j \neq i}\right\}_{i \in \mathcal{I}}+\sum_{i, j \in \mathcal{I}, j \neq i} \mu_{i j}\left(p_{j}-p_{i j}\right)
\end{aligned}
$$

$$
\text { subject to } p_{i} \in \mathcal{P}_{i}, p_{i j} \in \mathcal{P}_{j}, \quad \forall i, j,
$$

where $\lambda$ can be referred to as the power price in this case, and $\mu_{i j} \mathrm{~s}$ are the consistency prices for all $i, j$. Next, we decompose the Lagrangian into $\mathcal{I}$ sub-problems, one for each BS. The sub-problem for BS $i$ can be expressed as

$$
\begin{gathered}
\operatorname{maximize} s_{i} \\
p_{i},\left\{p_{i j}\right\}_{j \neq i} \\
\text { subject to } p_{i} \in \mathcal{P}_{i}, p_{i j} \in \mathcal{P}_{j},
\end{gathered}
$$

where $s_{i}$ can be viewed as a surplus function that takes into account the pricing terms, and is given by

$$
\begin{gathered}
s_{i} \triangleq U_{i}\left(p_{i},\left\{p_{i j}\right\}_{j \neq i}\right)-\lambda p_{i}+\left(\sum_{j \in \mathcal{I}, j \neq i} \mu_{j i}\right) p_{i} \\
-\sum_{j \in \mathcal{I}, j \neq i} \mu_{i j} p_{i j}
\end{gathered}
$$

Then, the master dual problem can be stated as:

$$
\begin{aligned}
& \underset{\left\{\lambda, \mu_{i j}\right\}}{\operatorname{minimize}} g\left(\lambda,\left\{\mu_{i j}\right\}_{j \neq i}\right) \\
& \text { subject to } \lambda \geq 0,
\end{aligned}
$$

which can be easily solved by the gradient method, using the following iterates for updating the dual variables:

$$
\lambda(n+1)=\left[\lambda(n)-\alpha\left(P_{\max }-\sum_{i \in \mathcal{I}} p_{i}\right)\right]^{+},
$$

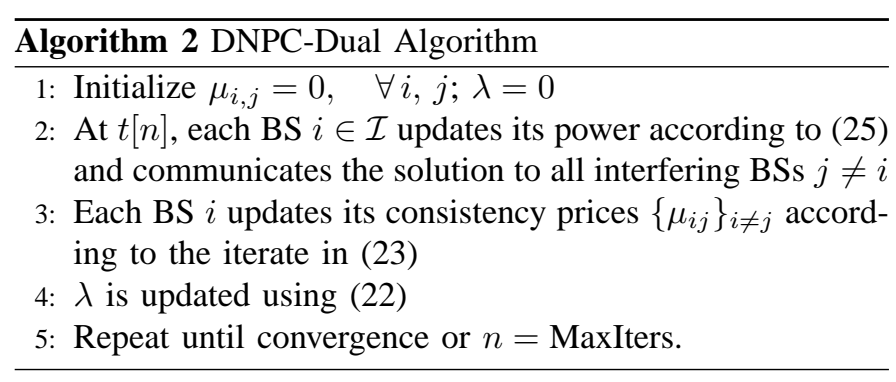

$$
\mu_{i j}(n+1)=\mu_{i j}(n)-\alpha\left(p_{j}-p_{i j}\right) \quad \forall i, j .
$$

A similar iterative procedure can be employed for solving (19) as well, where BS $i$ would step in direction of gradient (projected on local constraint set) to increase the objective over $p_{i}$ and $p_{i j}$. The gradient can be expressed as

$$
\nabla s_{i} \triangleq\left\{\begin{array}{l}
\nabla_{p_{i}} s_{i}=\pi_{i i}+\sum_{j \in \mathcal{I}, j \neq i} \mu_{j i} \\
\nabla_{p_{i j}} s_{i}=\pi_{i j}-\mu_{i j} \quad \forall j \in \mathcal{I}, j \neq i,
\end{array}\right.
$$

where $\pi_{i i}$, and $\pi_{j i}$ are defined in (14) and (15), respectively. Thus, the gradient projection update rule for BS $i \in \mathcal{I}$ is given by

$$
\begin{aligned}
p_{i}(n+1) & =\left[p_{i}(n)+\beta \nabla_{p_{i}} s_{i}\right]_{\mathcal{P}_{i}}, \\
p_{i j}(n+1) & =\left[p_{i j}(n)+\beta \nabla_{p_{i j}} s_{i}\right]_{\mathcal{P}_{j}}, \quad \forall j,
\end{aligned}
$$

where $[\bullet]_{\mathcal{X}}$ denotes the projection on set $\mathcal{X}$, and $\beta$ is the scaling factor for the gradient. Therefore, in a given iteration each BS $i \in \mathcal{I}$ updates not only the consistency prices $\mu_{i, j}$ and $\lambda$, but also its own power $p_{i}$, and auxiliary powers $\left\{p_{i j}\right\}_{j \neq i}$. The algorithm is summarized as Distributed Network Power Control with Dual decomposition (DNPC-Dual) in Algorithm 2. Note that $\lambda$ updates are based on global knowledge of the transmit powers, and thus this algorithm is not fully decentralized.

\section{Numerical Results}

In this section, we analyze the performance of the proposed algorithms and compare them with various cooperative and non-cooperative approaches. The simulation scenario is presented first, followed by the discussion of results.

\section{A. Simulation Scenario}

We consider a small cell network scenario, comprising of multiple low power BSs, serving a number of MSs deployed in a multistory building. Figure 1 shows the simulation scenario, with an illustration of the BS locations inside the building. The propagation characteristics inside the buildings are modeled according to a WINNER A1 office model [9]. The average path loss is

$$
\mathrm{PL}[\mathrm{dB}]=A \log _{10}(d)+B+C \log _{10}\left(\frac{f_{c}}{5}\right)+D L_{\mathrm{w}},
$$

where $d[\mathrm{~m}]$ is the distance between the transmitter and receiver, $f_{c}[\mathrm{GHz}]$ is the carrier frequency of the system, 


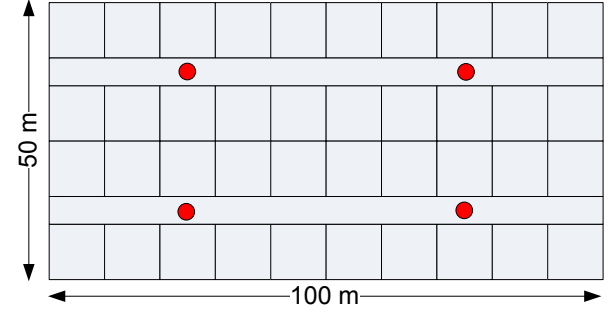

Fig. 1. Layout of the WINNER office building. The dots in the figure represent small-cell BSs.

TABLE I

WINNER II PATH LOSS MODEL

\begin{tabular}{ll}
\hline Building dimensions [m] & $100 \times 50$ \\
Room dimensions [m] & $10 \times 10$ \\
Corridor width [m] & 5 \\
Room height [m] & 3 \\
BS antenna height [m] & 2 \\
MS antenna height [m] & 1 \\
Number of floors & 3 \\
Antenna patterns & omni directional \\
Carrier frequency [GHz] & 2.6 \\
Line-of-sight & in same room/corridor \\
Path loss coefficients & $A=18.7, B=46.8, C=20$ \\
Inner wall loss [dB] & 5 (per wall) \\
\hline
\end{tabular}

$L_{\mathrm{w}}[\mathrm{dB}]$ identifies the (discrete) loss that is produced by walls (and windows), and $D$ is the number of walls between transmitter and receiver (see Table I for more details). Details of other system parameters are given in Table II. Each MS selects a single BS as a serving BS on the basis of received power in the downlink. It is assumed that a backhaul connection exists between the BSs, over which the exchange of prices takes place.

\section{B. Performance Analysis}

The performance of the proposed DNPC algorithms that solve the optimization problem given by (8) is compared for different cases, that entail varying degrees of cooperation among cells. For comparison, the (Cumulative Distribution Functions) CDFs of network utility are illustrated in Fig. 2, for 1000 random network instances generated according to the simulation parameters. To analyze the gain from pricing exchange, the baseline non-cooperative case considered is Equal Power, where the total network power is distributed equally among the BSs.

The DNPC-Primal algorithm is also considered without

TABLE II

SimUlation PARAMETERS

\begin{tabular}{ll}
\hline Number of BSs & 12 \\
Number of MSs & 12 \\
Maximum Transmit Power $\left(P_{\max }\right)[\mathrm{dBm}]$ & 20 \\
Minimum Transmit Power $\left(P_{\min }\right)[\mathrm{dBm}]$ & -10 \\
Noise figure [dB] & 9 \\
Thermal noise [dBm/Hz] & -174 \\
Shadow fading correlation & 0.5 \\
Shadow fading standard deviation [dB] & 3 \\
\hline
\end{tabular}

TABLE III

CONVERgENCE OF DNPC Algorithms

\begin{tabular}{|c||c|c|}
\hline & Converged Instances (\%) & Iterations (mean) \\
\hline DNPC-Primal & 99.70 & 70.20 \\
\hline DNPC-Dual & 99.10 & $8.93 \times 10^{3}$ \\
\hline DNPC-Primal(w/o Pri) & 100 & 7.63 \\
\hline
\end{tabular}

power prices, i.e. DNPC-Primal (w/o Pri). In this case, for all BSs $i$, the $D_{i}$ comprises of the benefit terms only. Note that it is a partially cooperative scheme, as each BS $i$ reports its $D_{i}$ to all BSs $j \neq i$. On the other hand, in fully cooperative DNPCPrimal, each BS $i$ receives both $\left\{D_{j}\right\}_{j \neq i}$, and prices $\left\{\pi_{j i}\right\}_{j \neq i}$ from all BSs. The maximum power increase (or decrease) stepsize $\delta_{\max }$ is set to $1 \%$ of the current BS transmit power. Likewise, the dual decomposition (DNPC-Dual) is also considered with $\alpha=0.45$ and $\beta=1$. It is observed to be extremely sensitive to the step-size related parameters involved, and for most cases it requires a large number of iterations to converge. It can be seen that a substantial gain of the order of $4.5 \times$ in terms of median network utility can be achieved over the baseline Equal Power case by both DNPC-Primal and DNPC-Dual algorithms. Some loss is observed for the DNPCPrimal (w/o Pri), which makes sense as the decisions on power changes are made by considering the partial information (i.e. only $\pi_{i i}$ terms). In Fig. 2, the corresponding user data-rates are shown with zoom on lower end of the CDF of user datarates, which highlights the benefit of PF-Rate utility, as it can be seen that data-rates of MSs experiencing low SINRs are increased significantly.

To study the convergence characteristics for DNPC-Primal, and DNPC-Dual, we use a formal convergence criterion that the difference between achieved network utility and the optimal utility is less than $5 \%$. It should be noted that the slight discrepancy observed in Fig. 3 between the rates achieved by DNPC-Primal and DNPC-Dual is due to this stopping criterion, as it is not very strict. Nevertheless, it has been chosen to speed-up the convergence of DNPC-Dual to some extent. From Fig. 3, we observe 100\% improvement over baseline case at $10 \%$-tile of CDF of user data-rates. Moreover, convergence is observed in almost all network instances (more than $99 \%$ with suitable update step-size parameters) for both algorithms, albeit with a very high number of iterations for DNPC-Dual. Therefore, the maximum number of iterations is set to MaxIters $=40 \times 10^{3}$. The convergence statistics are summarized in Table III.

\section{CONCLUSiONS}

In this paper, we proposed distributed pricing algorithms based on decomposition methods, for network utility maximization in $\mathrm{CR}$ networks under network-wide power constraint. Primal decomposition is used to design an online algorithm for PF-Rate maximization, while meeting the networkwide power constraint. Numerical simulations carried out in a practical small cell $\mathrm{CR}$ network scenario show that the proposed approach performs better than the non-cooperative, 


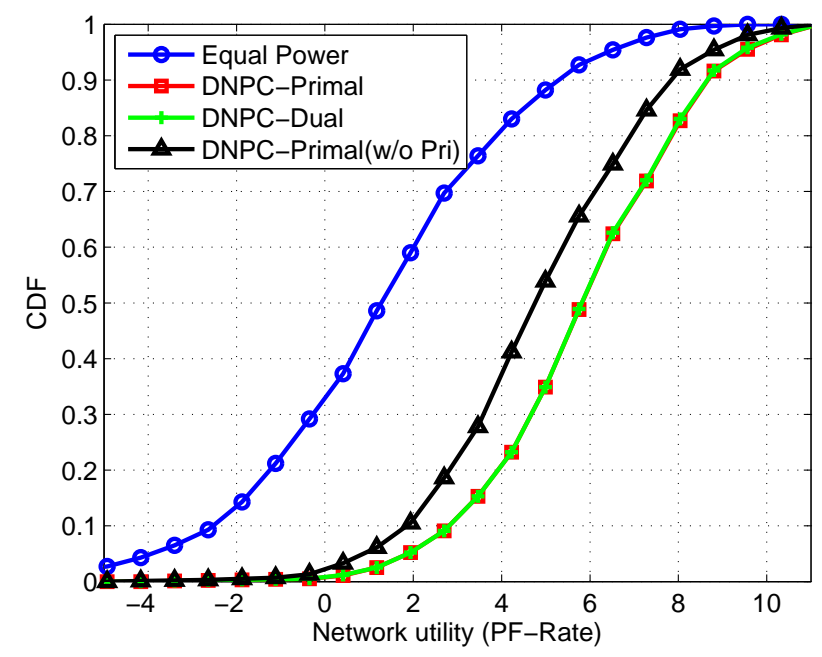

Fig. 2. Comparison of network utility for different cooperative and noncooperative power allocation schemes.

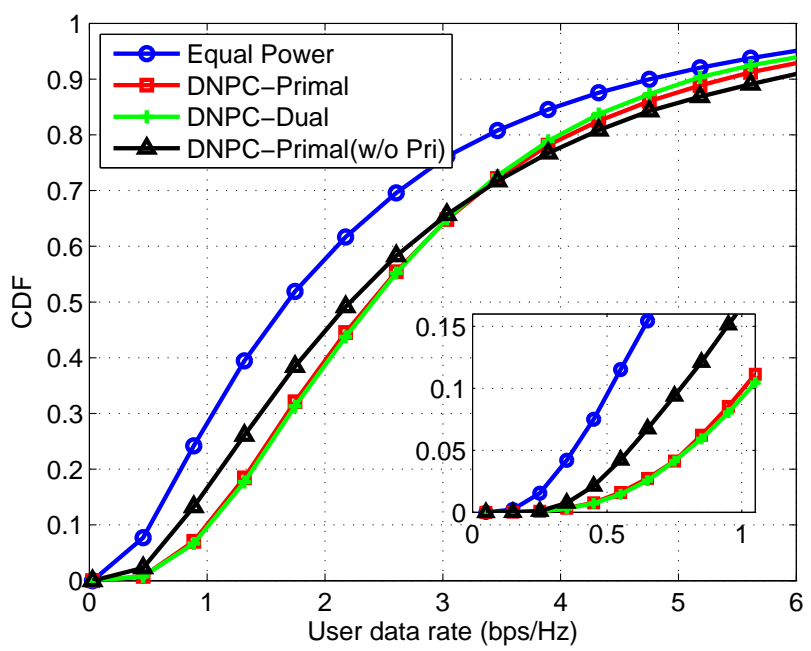

Fig. 3. Comparison of user data-rates for different cooperative and noncooperative power allocation schemes. The inset shows the lower end of CDF to clarify the gains achieved by the use of pricing in a small cell CR network.

and partial cooperative schemes, in terms of the network utility and the data-rates achieved by users.

\section{ACKNOWLEDGMENT}

This work was supported in part by the Academy of Finland, grant 257315, and the European Commission in the framework of the FP7 project ITC-317669 METIS.

\section{REFERENCES}

[1] M. Nekovee, "A survey of cognitive radio access to TV white spaces," Int. J. Digital Multimedia Broadcasting, pp. 11, Article ID 236568, Apr. 2010

[2] CEPT ECC, "Licensed shared access (LSA)," ECC Report 205, Feb. 2014.

[3] K. Koufos, K. Ruttik, and R. Jantti, "Controlling the interference from multiple secondary systems at the TV cell border," in Proc. IEEE Int. Symp. on Personal, Indoor and Mobile Radio Commun., Sep. 2011, pp. 645-649.

[4] J.-S. Pang, G. Scutari, D. Palomar, and F. Facchinei, "Design of cognitive radio systems under temperature-interference constraints: A variational inequality approach," IEEE. Trans. Signal Process., vol. 58, no. 6, pp. 3251-3271, Jun. 2010.

[5] S. Gong, P. Wang, and D. Niyat, "Optimal power control in interferencelimited cognitive radio networks," in Proc. IEEE Internat. Conf. Commun. Syst., Nov. 2010, pp. $82-86$.

[6] S.-J-Kim and G. Giannakis, "Optimal resource allocation for MIMO ad hoc cognitive radio networks," IEEE Trans. Inf. Theory, vol. 57, no. 5, pp. 3117-3131, May 2011.

[7] K. Koufos and R. Jantti, "Proportional fair power allocation for secondary transmitters in the TV white space," Journal of Electrical and Computer Engineering, pp. 1-8, 2013.

[8] E. Nekouei, H. Inaltekin, and S. Dey, "Throughput scaling in cognitive multiple access with average power and interference constraints," IEEE Trans. Signal Process., vol. 60, no. 2, pp. 927-946, Feb. 2012.

[9] P. Kyösti et al., "Winner II channel models," Winner II, Tech. Rep. D1.1.2 V1.2, 2007.

[10] F. Kelly, "Charging and rate control for elastic traffic," European Trans. Telecommun., vol. 8, pp. 33-37, 1997.

[11] Z.-Q. Luo and S. Zhang, "Dynamic spectrum management: Complexity and duality," IEEE J. Select. Areas Commun., vol. 2, no. 1, pp. 57-73, Feb. 2008.

[12] F. Ahmed, A. A. Dowhuszko, and O. Tirkkonen, "Distributed algorithm for downlink resource allocation in multicarrier small cell networks," in Proc. IEEE Int. Conf. on Commun.: Workshop on Small Cell Wireless Networks, Jun. 2012, pp. 6802-6808.

[13] D. Palomar and M. Chiang, "Alternative distributed algorithms for network utility maximization: Framework and applications," IEEE Trans. Autom. Control, vol. 52, no. 12, pp. 2254-2269, Dec. 2007.

[14] J. Huang, R. Berry, and M. Honig, "Distributed interference compensation for wireless networks," IEEE J. Select. Areas Commun., vol. 24, no. 5, pp. 1074-1084, May 2006.

[15] S. Boyd and L. Vandenberghe, Convex Optimization. New York, NY, USA: Cambridge University Press, 2004.

[16] D. Palomar and M. Chiang, "A tutorial on decomposition methods for network utility maximization," IEEE J. Select. Areas Commun., vol. 24, no. 8, pp. 1439-1451, Aug. 2006. 